\title{
INTEGRATED PROCESSING OF PHOTOGRAMMETRIC AND LASER SCANNING DATA FOR FRESCOES RESTORATION
}

\author{
N. Bruno ${ }^{1}$, S. Mikolajewska ${ }^{1}$, R. Roncella ${ }^{1}$, A. Zerbi ${ }^{1}$ \\ ${ }^{1}$ University of Parma, Department of Engineering and Architecture, Parco Area delle Scienze, 181/A, 43124 Parma, Italy- \\ (nazarena.bruno, sandra.mikolajewska, riccardo.roncella, andrea.zerbi)@unipr.it
}

\section{Commission II}

KEY WORDS: Integrated survey, Laser scanning, Photogrammetry, Cultural Heritage documentation, frescoes restoration.

\begin{abstract}
:
The integration of photogrammetry and Terrestrial Laser Scanner (TLS) techniques is often desirable for Cultural Heritage digitization, especially when high metric and radiometric accuracy is required, as for the documentation and restoration of frescoed spaces. Despite the many technological and methodological advances in both techniques, their full integration is still not straightforward. The paper investigates a methodology where TLS and photogrammetric data are processed together through an image matching process between RGB panoramas acquired by the scanner's integrated camera and frame imagery acquired through photographic equipment. The coregistration is performed without any Ground Control Point (GCP) but using the automatically extracted tie points and the known Exterior Orientation parameters of the panoramas (gathered from TLS data original registration) to set the ground reference. The procedure allowed for effective integrated processing with the possibility of take benefit from TLS and photogrammetry pros and demonstrated to be reliable even with low overlap between photogrammetric images.
\end{abstract}

\section{INTRODUCTION}

Numerous projects in recent years have shown how increasingly sophisticated surveying techniques and instruments can be a valuable support for Cultural Heritage digitization. Whatever the goal of such an operation (documentation, knowledge, dissemination, restoration, etc.), the integration of different techniques is desirable. The combination of image- and rangebased techniques allows digitization with high metric and radiometric accuracy. This is particularly useful in the field of restoration, where accurate documentation is required for quantitative and qualitative analysis of the surfaces state of conservation (Bevilacqua et al., 2016; Patrucco et al., 2020, Gabellone et al., 2020). These two issues are fundamental, for example, in frescoed spaces. In these contexts, accurate documentation is useful for the elaboration of diagnostic maps, for the study of surfaces that cannot be reached directly by operators (without scaffolding), for the simulation of restoration operations in a virtual space (before they are conducted in situ), and so on.

Despite the many technological and methodological advances in both techniques, which have made them almost independent from each other (e.g., laser scanner integrated cameras for RGB data acquisition, high automation in photogrammetric process), the advantages provided by their integration have been largely addressed in literature, with reference to different fields of application (Remondino, 2011; Grussenmeyer et al., 2012; Balletti et al., 2015; Bianchi et al., 2016; Luhmann et al., 2020). Traditionally, in most cases, these techniques are processed separately and then merged. There are numerous experiences of surveys performed with both methodologies and co-registered, using common Ground Control Points (GCP) or Iterative Closest Points (ICP) algorithms (Chiabrando et al., 2014; Valente et al., 2019; Bitelli et al., 2020).

Different methodologies and workflows have been developed to increase automation and accuracy (Rönnholm et al., 2007; Hassan et al., 2019), but the registration procedure is still not straightforward. For instance, recent software developments provided by Agisoft (Metashape v. 1.7) allow laser scanning data to be imported into the photogrammetric environment converted in spherical images with associated range data and processed simultaneously with images (Agisoft Helpdesk Portal). This capability allows the integrated processing and avoids extra data fusion steps. Examples on the introduction of this procedure in the processing workflow can be found in (Fiorillo et al., 2021).

The paper investigates a workflow where photogrammetric and Terrestrial Laser Scanner (TLS) data are processed simultaneously from the initial steps, without considering them as separate datasets to be merged. The co-registration of photogrammetric frame images on TLS data is performed without any Ground Control Point (GCP), and directly using the laser scan data as ground reference to set the object reference system.

The aim is to enhance the strengths of each survey methodology to obtain a unique $3 \mathrm{D}$ model in which the geometry is derived from TLS data and the radiometry from photogrammetric images. TLS permits to implement a simpler and more straightforward survey planning for the interiors survey, while the use of photogrammetric blocks allows obtaining higher quality orthophotos.

The methodology was tested on a part of the Monastery of San Paolo in Parma (Italy), where an accurate survey was required to document the state of conservation of the architectural complex and all the frescoes inside.

\section{CASE STUDY: THE CAMERA DI SAN PAOLO}

The museum itinerary known as "Camera di San Paolo", located inside the ancient Benedictine female monastery of St. Paolo in Parma, today includes nine rooms. Some of these spaces were once the private apartment of the abbess Giovanna from Piacenza. What makes this itinerary extraordinary is the presence of the so-called "Chamber of the Abbess", whose umbrella vault was frescoed in 1519 by Antonio Allegri, known as Correggio, with an illusionistic pergola decoration in which there are false oval openings from which groups of putti overlook. The lunettes 
on the vertical walls are then completed by a series of false monochrome niches that simulate the presence of statues. On the fireplace, the goddess Diana is represented, an evident reference to the abbess Giovanna, who had commissioned the work. Adjacent to the "Chamber of the Abbess" there is another important space, frescoed in 1514 by Alessandro Araldi. Here the pavilion vault with lunettes presents a rich grotesque ornamentation in which, in the centre, the artist opens a false circular opening with a balustrade from which musician putti appear.

As part of an extensive restoration project of the Monastery of San Paolo, the "Camera di San Paolo and Cella di S. Caterina Valorization Project" was signed in March 2021. One of the main goals of the plan concerns the documentation of the architectural complex state of conservation, with particular attention to the frescoes and the wooden and stone decorative apparatus. To this aim, an integrated survey campaign of the complex was started in June 2021. An accurate documentation of the museum itinerary spaces was essential for the elaboration of the architectural two-dimensional drawings with orthophotos of every wall and ceiling in a scale 1:50, required for the restoration operations carried out in July 2021, which aimed to guarantee the adequate conservation of the complex.

Although the survey campaign concerned all the rooms of the museum itinerary, this paper will outline the methodology with reference only to the two main rooms described above (Figure 1), the chamber frescoed by Araldi (hereinafter referred to as R1) and the "Chamber of the Abbess" (hereinafter referred to as R2).

\subsection{Data acquisition}

To document the architectural complex, both from metric and radiometric point of view, it was decided to carry out a TLS and photogrammetric integrated survey.

TLS is particularly suitable for surveying interior spaces with complex geometry and articulated spatial distribution, as those in this case study. In this specific context, the TLS survey results more advantageous in terms of time for data acquisition, robust data registration and management. On the other hand, the photogrammetric survey guarantees a better radiometric accuracy, but normally would entail longer data acquisition and processing times and would require a topographical survey to set the ground reference. In this case study, the whole operation would have been further complicated by the impossibility of applying targets on the frescoed surfaces.

The TLS acquisitions of the whole building were performed in one day. A Leica RTC360 laser scanner was used. The instrument is equipped with a 36 Mpixel HDR camera and, for each scan, acquires a high-resolution spherical image (panorama coregistered with the range image) by stitching 36 single images of $4000 \times 3000$ pixel resolution. Panoramas are generally employed to get the colours of the $3 \mathrm{D}$ point cloud but can be exported as equirectangular images. However, by their nature, spherical images might have a very variable image scale, this makes the TLS data not optimal for providing high quality orthophotos. Particular attention was paid to the choice of station points. To ensure adequate documentation of the rooms, reducing as much as possible the occlusions but, at the same time, limiting the number of scans (for optimal efficiency) were considered a fundamental requirement.

A total of 41 scans with the corresponding panoramas were acquired, including one to three scans per room (depending on its dimension, spatial complexity, and decorative apparatus) and one scan for each passage, to ensure a proper registration. R1 and R2 were surveyed with 3 scans each.

The acquired data was then registered in a unique reference system, allowing to obtain a unique point cloud of the entire complex (Figure 1). Thanks to the relatively small size of the rooms and a distance between the laser scanner and the object always lower than $6 \mathrm{~m}$, a resolution of $6 \mathrm{~mm}$ at $10 \mathrm{~m}$ distance was considered adequate.

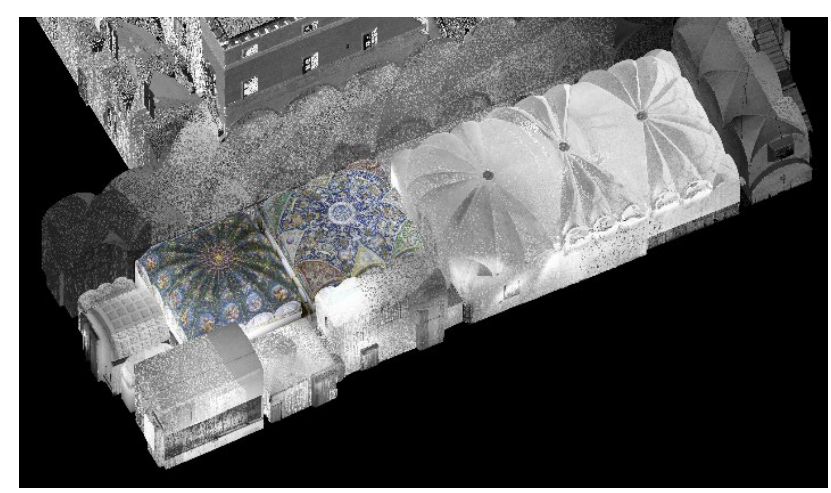

Figure 1. The complete point cloud after registration. The two rooms investigated in this paper (R1 and R2) are represented with RGB colours. $\mathrm{R} 1$ is on the right of the image and $\mathrm{R} 2$ on the left.

In addition, for each room, a close-range photogrammetric survey was performed using a full-frame Nikon D3X DSLR camera (6048x 4032 pixels resolution and 6 micrometres pixel size) equipped with a $35 \mathrm{~mm}$ Nikkor optic or with a $24 \mathrm{~mm}$ Nikkor optic for the smaller rooms. This campaign was conducted in several days. The camera was mounted on a tripod and the images were acquired using a wired remote control, to stabilize the sensor and avoid blurring, which is possible in indoor spaces with non-optimal lighting conditions. To ensure uniformity from a radiometric point of view, all photographs were taken under artificial light conditions and using a colour calibration chart.

As far as R1 and R2 are concerned, the vertical walls were surveyed with three horizontal strips per side, joined through oblique images framing the corners. The vaults were acquired with two strips at different height per side and additional diagonal images. A total of 167 and 171 images were collected for the two rooms respectively, with an average Ground Sampling Distance (GSD) equal to $0.7 \mathrm{~mm} / \mathrm{pix}$.

\section{DEVELOPED METHODOLOGY}

\subsection{Integrated data processing}

The processing was performed using the software Agisoft Metashape, thanks to its capability of importing structured point cloud data.

The raw laser scans of the entire complex were registered using the software Leica Cyclone and exported as structured point cloud data in ASTM E57 format, jointly with the corresponding equirectangular panoramas (resolution $8192 \times 4096$ pixel). For each room, the.$e 57$ point clouds were imported into Metashape. The software performs an initial pre-processing where the structured point clouds are converted into .tif equirectangular images, where each pixel corresponds to a scan point. For each pixel, the radiometric data (RGB, if acquired, the intensity otherwise) and the depth value (stored in the image depth map) are then saved. Once imported, TLS data are RGB spherical panoramas with corresponding depth map and known Exterior Orientation (EO) parameters (principal point location and camera rotation) as provided by the initial TLS data registration. Integrating the close-range photogrammetric survey, therefore, image and TLS data registration results in a standard image matching process between spherical and frame images (i.e.,the 
orientation process jointly involved the two datasets of equirectangular and D3X frame images). The EO parameters of the equirectangular images have been kept fixed and used as reference, while the EO parameters of the frame images have been computed during the Bundle Block Adjustment (BBA) based on the corresponding tie points between images and spherical panoramas. With this procedure, the original registration of the TLS data is preserved and used as reference, while the frame images are registered with the panoramas, without the need of any Ground Control Point (GCP).

As far as the interior orientation is concerned, the equirectangular images have been processed considering a spherical camera model, while for the frame images a self-calibration was performed during the BBA. The extraction of tie-points was performed considering images at full resolution ("high quality accuracy" in Metashape terminology).

The equirectangular images produced by Metashape are obtained from the coloured TLS point cloud, so they are not a real image and are affected by any imperfections of the point cloud. This, for example, is particularly visible on corners, where the laser beam does not capture the edge perfectly, which turns in no data on the panorama. To improve the matching accuracy, the equirectangular images obtained from the coloured TLS point cloud have been replaced with the panoramas captured directly by the RTC360 built-in camera since, generally, they have higher quality and resolution.

After the orientation process, the mesh model has been directly computed using only the depth maps associated to the equirectangular images and provided by the TLS data, (i.e., triangulating the laser scanner survey). This mesh model has been used as Digital Surface Model (DSM) for the subsequent generation of orthophotos, while the radiometric information has been provided by Nikon D3x images only.

\subsection{Frame image block orientation accuracy}

In the proposed methodology, the co-registration between TLS and photogrammetry data is performed through an image matching process. Compared to a standard photogrammetric pipeline, this approach involved an unusual ground control solution based on the known panorama's EO parameters derived from TLS scans registration. The frame images orientation is therefore affected by the integration of different camera models (pinhole and spherical) and by different image resolutions and Ground Sampling Distance (GSD), which for the panoramas is much lower than for frame images $(0.7 \mathrm{~mm} /$ pix versus 4.16 $\mathrm{mm} /$ pix for R1 and $4.37 \mathrm{~mm} /$ pix for R2).

A first validation of the methodology is to evaluate the internal consistency of the block (considered as the collinearity equation residuals) and the accuracy achievable using such ground control solution. The results have been compared to those provided using a standard procedure where the same dataset of frame images is oriented using GCP.

For each room, three different blocks have been processed and compared. Table 1 summarizes the characteristics of each block and the different ground control solutions adopted.

\begin{tabular}{|l|l|l|l|}
\hline Block & Images & Constraints & GCP precision \\
\hline A & Frame & 15 GCP & $0.005 \mathrm{~m}$ \\
\hline B & Frame & 10 GCP & $0.005 \mathrm{~m}$ \\
\hline C & $\begin{array}{l}\text { Frame } \\
\text { TLS Panoramas }\end{array}$ & $\begin{array}{l}\text { Panoramas } \\
\text { OE }\end{array}$ & $/$ \\
& & & \\
\hline
\end{tabular}

Table 1. Parameters used for orientation process.

Block A and Block B differ in the strength of the control network, using a different number of GCP. The use of a greater number of GCP makes the orientation solution generally more stable and reliable. Block $\mathrm{C}$ was instead processed according to the developed methodology, processing together frame images and TLS panoramas, without GCP.

Due to the characteristics of the building and the artistic value of the frescoes, no target was allowed to be installed on the walls. Therefore, GCP were selected on architectural features, e.g, edges, corners, or well-recognizable colour changes on the frescoes. To ensure uniformity in the comparison between the methodologies, the coordinates of the GCP have been extracted from the TLS point cloud.

The accuracy of each block was evaluated analysing: (a) the reprojection residuals of collinearity equations computed on the tie points extracted during the BBA, to assess the internal consistency of the photogrammetric block. (b) The residuals on Check Points (CP), to have an evaluation of the accuracy of the final object reconstruction. It is worth noting that, for uniformity, the same $\mathrm{CP}$ were considered in the stats and the image coordinates of both $\mathrm{CP}$ and GCP were the same for the three blocks. (c) The distance between the mesh model obtained from TLS data (used as reference) and the photogrammetric DMS resulting from the dense matching between frame images only. Two different comparisons were made: in the former the DMS were further co-registered through an ICP algorithm, to avoid systematic differences, in the latter no further alignment was performed, to evaluate the consistency between the two datasets derived only from matching.

\subsection{TLS data and frame images co-registration assessment}

A second evaluation focused on the agreement between TLS and photogrammetric data. It is very important especially when they are used together, as in the generation of orthophotos where the DSM is derived from the TLS and the images from the photogrammetric survey.

The assessments involved the geometrical correspondence between orthophotos obtained from different images, computed in the overlapping areas.

The comparison between the orthophotos was carried out by selecting, for each wall and vault inside the rooms, pairs of overlapping images. For each pair, the two orthophotos corresponding to each image were generated, using the same DSM, projection plane, resolution, and boundaries. In this way, excluding relative orientation errors, the orthophotos should be identical in the overlap part.

The correspondence was computed through a feature-based method using Matlab functions. The choice of preferring a feature-based to an area-based method, which would have allowed a pixel-to-pixel comparison between the images, was made (i) to avoid that slight chromatic variations can be erroneously considered as displacements, (ii) to calculate displacements mainly on the areas with more textures (such as the frescoed areas). The two orthophotos are obtained from two different images, so, despite the use of a colour calibration chart, they may have slight colour variations due to different exposure or illumination conditions that should not be considered as shifts. In addition, the importance of image co-registration error is significant on surfaces with a lot of texture or discontinuities, where co-registration errors can result in evident irregularities in the orthophoto. Conversely, on continuous and uniform surfaces, such as plastered surfaces, the consequences of an inaccurate orthorectification are less important.

Figure 2 shows the steps of the implemented procedure for comparison: a) The orthophotos have been initially converted from RGB to grayscale and cropped to limit the comparison to the overlapping area; b) to extract interest points from images, 

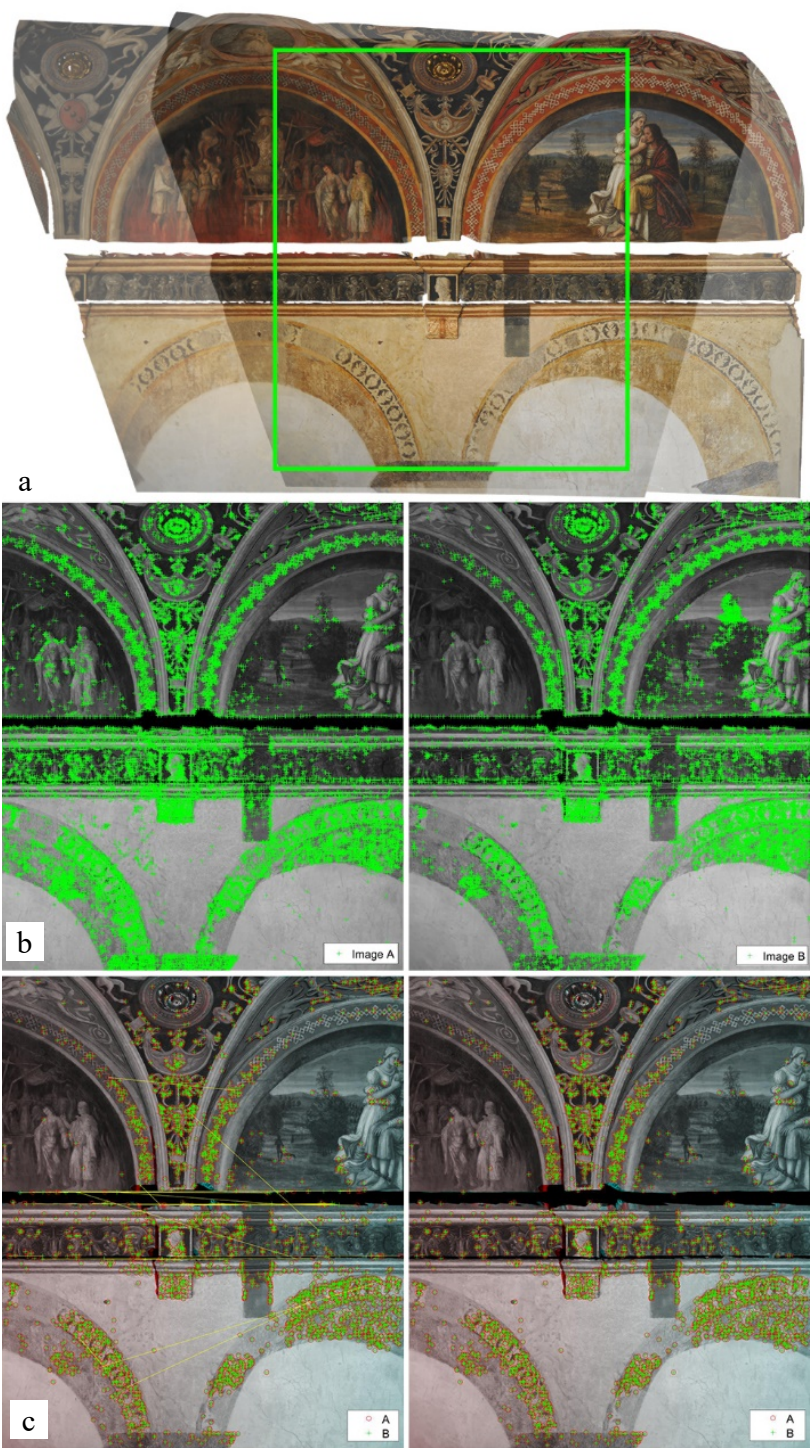

Figure 2. Procedure for orthophoto comparison. a) Orthophotos overlapping and cropping. b) Feature extraction from the two images. c) Feature matching and removal of outliers.

several algorithms were tested, including minimum eigenvalue algorithm (Shi and Tomasi, 1994), Speeded-Up Robust Features (SURF) algorithm (Bay et al., 2006) and Features from Accelerated Segment Test (FAST) algorithm (Rosten and Drummond, 2005). The use of minimum eigenvalue algorithm has provided the best performance. Corresponding features extracted on the two images were matched using their descriptors (Muja and Lowe, 2012), and the distance in pixels between the matched features was computed. To remove the matching outliers, a threshold on this distance has been set, based on the accuracy of the orientation. At the end, the Root Mean Square (RMS) distance value of all the valid matches was computed.

The analysis involved the Blocks $\mathrm{C}$ for the two rooms and, to have a term of comparison with the solution resulting from the traditional methodology, it was also carried out on the Blocks B, since the lower number of GCP used to constrain the orientation makes this solution more comparable.

\subsection{Assessment of frame image block strength on orthophoto accuracy}

A further analysis concerned the processing of datasets containing a different number of frame images, to evaluate the effect of image multiplicity and overlap. The purpose is to assess how much the internal strength of the frame image block affects the computation of EO and the final accuracy.

The analysis was carried out on the frescoed vaults of the two rooms. The vaults are the most significant and interesting elements but, at the same time, the most complex to survey. In addition, the presence of the frescoes, which represent a well contrasted texture, makes them particularly suitable for testing and validating the methodology.

For each room, seven different datasets with a progressively decreasing number of images were considered and processed according to the developed methodology, up to the coregistration of a single image on the TLS spherical images (Figure 3).

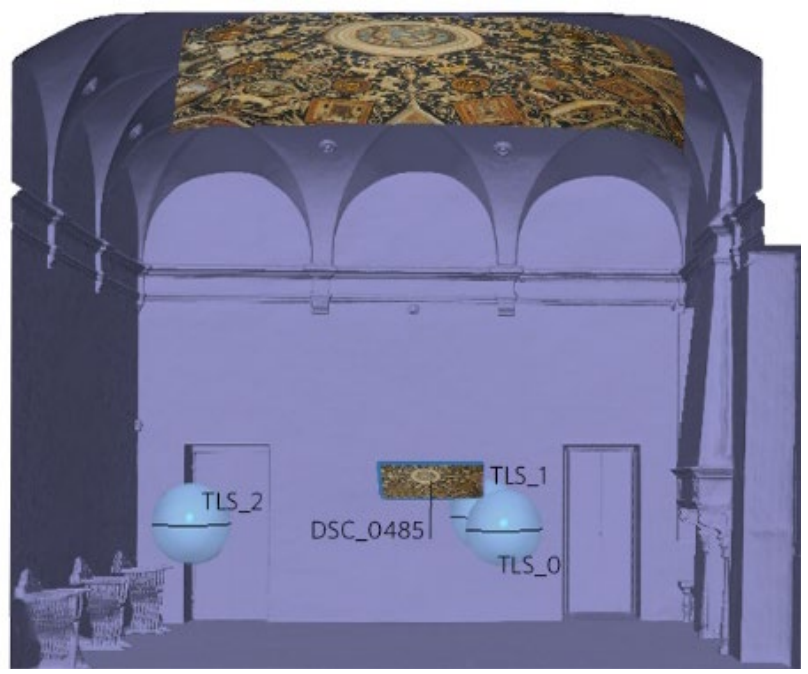

Figure 3. Example of co-registration of a single frame image on the spherical images from TLS (Room 1).

A first processing (referred to as Block D1) accounts for all the images framing the vaults excluding the ones framing the vertical walls. Then subsequent evaluated blocks are composed of one half of the images, one third and so on, progressively $\left(1 / 4^{\text {th }}\right.$ images, $1 / 6^{\text {th }}$ and $\left.1 / 8^{\text {th }}\right)$, until a single image was processed.

The accuracy evaluation the of these blocks was performed in comparison with Block $\mathrm{C}$, since it was processed using the same methodology and accounting for all the images acquired in the survey, including the walls, which contribute to strengthen the network geometry.

Statistics were obtained by analysing the residual of collinearity equations, the residuals on $\mathrm{CP}$ and the geometric differences between the orthophotos of the entire vault obtained from these different datasets and the one obtained from Block $\mathrm{C}$ (computed using the Matlab function just described).

\section{RESULTS}

\subsection{Frame image block orientation accuracy}

Table 2 shows the stats on collinearity, CP residuals and DSM comparisons, computed according to the methodology explained in Section 3.2.

The analysis of reprojection residuals shows that when panoramas are added to the process, the collinearity between frame images worsens. It can be observed that reprojection residuals increase by $40 \%$ and $35 \%$ for $\mathrm{R} 1$ and $\mathrm{R} 2$, respectively. This depends on the quality of the matching between frame and spherical images. The separate analysis of the reprojections of tie 


\begin{tabular}{|l|c|c|c|c|c|c|}
\hline & \multicolumn{3}{|c|}{ Collinearity } & & \multicolumn{2}{c|}{ DSM st. dev } \\
\hline Block & $\begin{array}{c}\text { Tie } \\
\text { Points } \\
{[\mathrm{n}]}\end{array}$ & $\begin{array}{c}\text { Proj. } \\
{[\mathrm{n}]}\end{array}$ & $\begin{array}{c}\text { Reproj } \\
\text { Resid. } \\
{[\text { [pix] }}\end{array}$ & $\begin{array}{c}\text { RMS } \\
\text { CP } \\
{[\mathrm{mm}]}\end{array}$ & $\begin{array}{c}\text { No } \\
\text { ICP } \\
{[\mathrm{mm}]}\end{array}$ & $\begin{array}{c}\text { ICP } \\
{[\mathrm{mm}]}\end{array}$ \\
\hline R1-A & 141722 & 630001 & 0.514 & 4.73 & 5.14 & 4.88 \\
R1-B & 125238 & 623068 & 0.534 & 5.10 & 4.99 & 4.57 \\
R1-C & 125320 & 629627 & 0.724 & 4.99 & 4.22 & 4.22 \\
R2-A & 128729 & 684441 & 0.569 & 5.32 & 3.50 & 3.43 \\
R2-B & 129866 & 686967 & 0.569 & 5.57 & 3.53 & 3.43 \\
R2-C & 129899 & 692021 & 0.765 & 4.56 & 3.46 & 3.46 \\
\hline
\end{tabular}

Table 2. Stats on collinearity, CP residuals and DSM comparison. 24 and $22 \mathrm{CP}$ are used for R1 and R2 respectively.

points between frame images only, frames and panoramas only, and panoramas only, shows that, despite the low number of tie points between frames and panoramas, they have the highest reprojection residuals. In detail, for both the rooms, among all the tie points almost the $95 \%$ is extracted between frame images, the $5 \%$ between frame and panoramas and the remaining between the panoramas. The formers have an average reprojection residuals equal to 0.391 pixels and 0.414 pixels for $\mathrm{R} 1$ and $\mathrm{R} 2$ respectively, the latter equal to 1.276 pixels and 1.363 pixels.

This may derive from the use of two different camera models, from the lower resolution of panoramas and from the peculiarity of the equirectangular projection that produces high deformations especially in the areas closer to the poles. In an indoor environment, this deformation is significant at the ceiling. The analysis of the reprojection residuals of each frame image shows that the highest RMS values are associated to the images related to the vault, particularly those depicting its ridge and matching the most deformed part of the equirectangular images. In these cases, the reprojection residuals are up to 1 pixel, while the mean values obtained for the overall Blocks $C$ are 0.721 and 0.765 for $\mathrm{R} 1$ and $\mathrm{R} 2$ respectively (Table 2 ).

It is worth noting that, although the number of images in the Blocks $\mathrm{C}$ is higher, the number of the extracted tie points is in line with the Blocks B. This may be related to the lower internal consistency of these blocks (with respect to Blocks A), which leads to considering some of the key points as invalid and excluding them from matching.

However, despite the noticeable deterioration of the internal coherence of the block, this does not result in macroscopic errors in the reconstruction of the $3 \mathrm{D}$ points. Residuals on $\mathrm{CP}$ (measured, for uniformity, exclusively on the frame images in the number of 24 for R1 and 22 for R2) do not reflect the behaviour identified by collinearity. On the contrary, in R2, Block C presents the smallest differences, while in R1 the behaviour of the three blocks is pretty the same.

From this analysis it is observed that, if the coordinates of the GCP are obtained from the TLS point cloud, a higher number of GCP is necessary to improve the accuracy. On the contrary, the matching between panoramas and frame images directly allows the accurate reconstruction of points on the object.

DSM comparison confirms this behaviour and also in this case the differences are quite low. However, it is noticeable how the orientation of the frame images using the panoramas as ground reference, directly removes the systematisms that are present, in particular in the R1 blocks. This result is particularly significant when, as in this case study, the two datasets (photogrammetric and laser) must be processed together, for example to obtain orthophotos. Here the accuracy of the co-registration between TLS model and frame images is crucial and must be achieved directly during the matching phase.

\subsection{TLS data and frame images co-registration assessment}

The good consistency of the co-registration between frame images and TLS model is also confirmed by the analysis performed on the overlapping orthophotos obtained from different images. Table 3 shows the RMS of distances between corresponding pixels on the orthophotos. For each room, the average RMS of each wall and vault is reported, to assess whether portions of the block suffer from greater inconsistencies (e.g., on the vault in view of the higher reprojection errors observed before).

For Block $\mathrm{C}$ the orthophotos were produced using the DSM obtained from the laser scanner data, while for Block B using the one obtained from the photogrammetric process, thus perfectly consistent with the oriented images. All the orthophotos were generated at a resolution of $1 \mathrm{~mm} / \mathrm{pix}$ and, in the comparisons, the matching outliers were filtered by applying a distance threshold of 3 pixel $(=3 \mathrm{~mm})$, which was deemed suitable in comparison to the reprojection residuals analysed in Table 2 .

\begin{tabular}{|c|c|c|}
\hline & \multicolumn{2}{|c|}{ RMS distance } \\
\hline Wall/Vault & $\begin{array}{c}\text { Block C } \\
{[\mathrm{mm}]}\end{array}$ & $\begin{array}{c}\text { Block B } \\
{[\mathrm{mm}]}\end{array}$ \\
\hline R1- Wall 1 & 0.405 & 0.337 \\
\hline R1- Wall 2 & 0.392 & 0.395 \\
\hline R1- Wall 3 & 0.412 & 0.384 \\
\hline R1- Wall 4 & 0.465 & 0.494 \\
\hline R1- Vault & 0.511 & 0.400 \\
\hline mean & 0.437 & 0.402 \\
\hline R2- Wall 1 & 0.440 & 0.372 \\
\hline R2- Wall 2 & 0.423 & 0.351 \\
\hline R2- Wall 3 & 0.486 & 0.480 \\
\hline R2- Wall 4 & 0.415 & 0.365 \\
\hline R2- Vault & 0.488 & 0.434 \\
\hline mean & 0.459 & 0.411 \\
\hline
\end{tabular}

Table 3. Orthophoto comparison.

From the analysis, it is observed that, in general, the geometric correspondence between the orthophotos is good and on average about $0.4 \mathrm{~mm}$, thus agrees with the reprojection residuals. For architectural applications this displacement is negligible and in line with the accuracy expected from the survey.

The differences on the Blocks B are generally slightly lower, but the analysis confirms the good consistency between the TLS model and the frame images.

Looking into the individual portions of the block, in room 2, the behaviour is homogeneous, while in room 1 , as expected, higher RMS values can be observed for the vault. This result agrees with the higher re-projection residuals resulting for these images, probably due to difficulties in matching with panoramas. In this room, the vault is also the area with the biggest differences compared to Block B.

\subsection{Assessment of frame image block strength on orthophoto accuracy}

Table 4 shows the statistics on the collinearity residuals and on the comparisons between orthophotos obtained by processing a progressively reduced number of images. The blocks named " $\mathrm{C}$ vault" are the same Blocks $\mathrm{C}$ described in Section 4.1, but, here, the statistics refers only to the images that frame the vault. These blocks were used as reference. The Blocks named D instead indicate blocks with reduced number of images. The number after D refers to the fraction of image reduction. Finally, D0 is the block with only one image. 

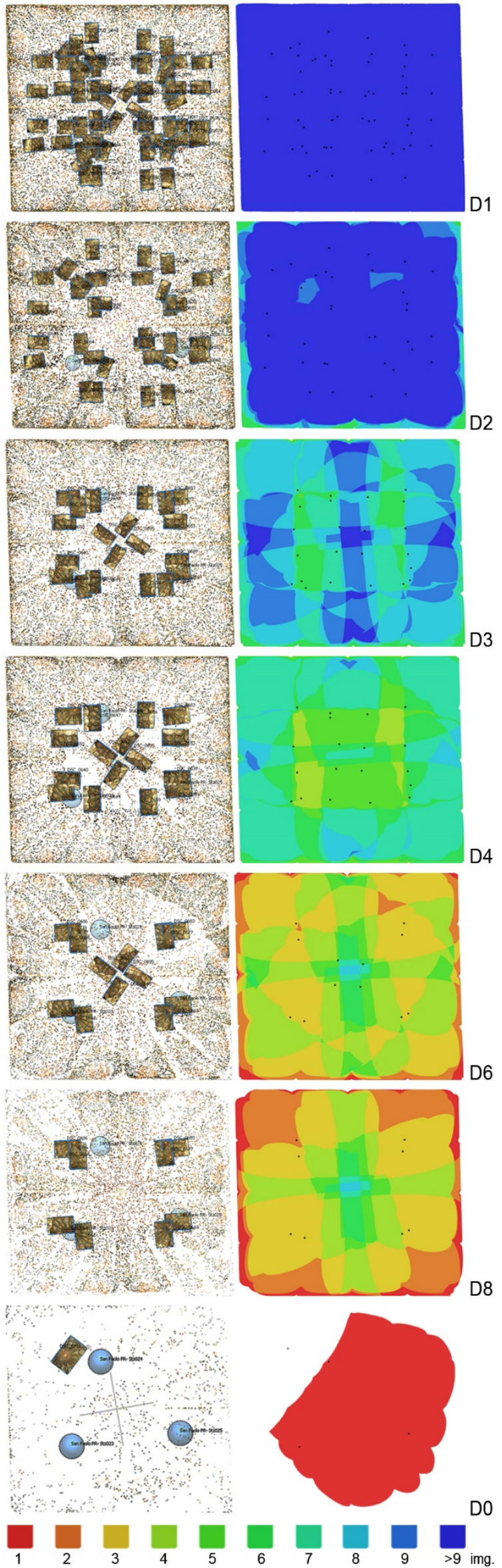

Figure 4. Block geometry and image overlap for the assessment of image multiplicity effect. Images refer to Room 2.

\begin{tabular}{|l|c|c|c|c|c|}
\hline Block & $\begin{array}{c}\text { Frame } \\
\text { img. } \\
{[\mathrm{n}]}\end{array}$ & $\begin{array}{c}\text { CP } \\
{[\mathrm{n}]}\end{array}$ & $\begin{array}{c}\text { Reproj } \\
\text { residuals } \\
\text { [pix] }\end{array}$ & $\begin{array}{c}\text { RMS } \\
\text { CP } \\
{[\mathrm{mm}]}\end{array}$ & $\begin{array}{c}\text { RMS } \\
\text { ortho. } \\
{[\mathrm{mm}]}\end{array}$ \\
\hline R1-C & 58 & 16 & 0.901 & 4.00 & - \\
Vault & 58 & 16 & 0.904 & 4.94 & 1.577 \\
R1-D1 & 30 & 16 & 1.060 & 6.04 & 2.141 \\
R1-D2 & 20 & 16 & 0.960 & 5.28 & 1.543 \\
R1-D3 & 16 & 16 & 0.905 & 5.28 & 1.668 \\
R1-D4 & 16 & 16 & 0.974 & 5.55 & 1.672 \\
R1-D6 & 12 & 16 & 0.978 & 6.55 & 2.486 \\
R1-D8 & 8 & - & 0.788 & - & 4.037 \\
R1-D0 & 1 & & & & \\
R2-C & & 7 & 0.909 & 5.18 & - \\
Vault & 61 & 7 & 0.937 & 3.86 & 1.261 \\
R2-D1 & 61 & 7 & 0.942 & 4.21 & 1.617 \\
R2-D2 & 32 & 7 & 0.936 & 4.05 & 1.638 \\
R2-D3 & 20 & 7 & 0.854 & 3.84 & 1.605 \\
R2-D4 & 16 & 7 & 0.817 & 4.83 & 2.238 \\
R2-D6 & 12 & 7 & $(5.25)$ & 2.707 \\
R2-D8 & 8 & 4 & 0.804 & 6.08 & 4.888 \\
R2-D0 & 1 & - & 0.916 & - &
\end{tabular}

Table 4. Stats on collinearity residuals and comparison between orthophoto. In brackets the stats computed on Block R2-D6 using only $4 \mathrm{CP}$ are provided for uniformity with the Block R2-D8.

The number of CP is different between the two rooms because, in R2 the identification of numerous architectural reference points on the top part of the vault has been difficult.

As for the orthophoto comparison, in this analysis the orthophotos of the entire vault derived from each block D were compared to the orthophoto obtained from Block C. All the orthophotos have a resolution of $1 \mathrm{~mm} /$ pix and were computed using the same DSM obtained from TLS data. The distance threshold for the matching outlier removal in this case was set at 10 pixels, to take into account that the comparison is made between orthophotos gathered from different blocks which have residuals on $\mathrm{CP}$ positions of $5 \mathrm{~mm}$ (on average).

From the results of the comparison between "C-vault" blocks and D1 blocks, it can be seen that processing a single portion of the block (in this case the vault) does not result in a reduction in accuracy due to the lower inner rigidity. The reprojection residuals of these blocks are similar and around 0.9 pixels, which probably result from the weakness of matching between panoramas and frames in the polar regions of panoramas. In R1 the residual on $\mathrm{CP}$ increases, while in $\mathrm{R} 2$ the behaviour is the opposite. The comparison of orthophotos, instead, shows differences of 1.5 and 1.2 pixels for the two rooms, respectively. By progressively reducing the number of images, the results remain quite stable as long as each area of the vault is framed by at least 3 images (Figure 4). On the other hand, when many areas are detected by 2 images only, the deviations increase to about 2.5 pixels ( $+58 \%$ in $\mathrm{R} 1$ and $+115 \%$ in $\mathrm{R} 2)$. As an extreme case, even a single image can be oriented on panoramas, but the results are $156 \%(\mathrm{R} 1)$ and $288 \%(\mathrm{R} 2)$ worse than in Blocks D1, and $62 \%$ (R1) and $80 \%(\mathrm{R} 2)$ worse than in Blocks D8, where there are many areas detected by only two overlapping images.

Therefore, from the results it is possible to infer that the resolution of the panoramas is fundamental for the matching accuracy and, thus, for the correct co-registration of the frame images. As long as the block of frame images has its internal strength, this effect is mitigated, otherwise, when the strength of the block is lost (up to the extreme case of the processing of a single image) the resolution of the panorama determines the uncertainty of the result. 


\section{DISCUSSION}

The implemented methodology has proven to be reliable, providing a proper co-registration between TLS data and photogrammetric images (Figure 5). The ground reference solution without the use of GCP makes it particularly suitable for surveys where the target installation is prevented or when an accurate topographical survey is not feasible. In addition, this procedure frees photogrammetry from the need for a topographical control survey, which usually represents a timeconsuming operation.

As highlighted in relation to reprojection residuals and tested on orthophoto, the resolution of the spherical image is crucial for adequate matching accuracy. A first operational recommendation for the use of this methodology is to acquire images at the maximum resolution allowed by the scanner and replace the panoramas generated by Metashape during the pre-processing of point cloud with the original spherical images acquired by the scanner's built-in camera.

In addition, since the integrated processing results in an image matching procedure, it is desirable that the frame images and the panoramas acquired by the TLS have similar radiometric characteristics, in order to improve the matching solution. To this end, a further investigation could concern the use of the raw images acquired by the TLS to generate the panorama with the desired exposure. The scanner performs a multi-exposure HDR capture and then combines several different exposures in the output panorama. Nevertheless, as can be seen from Figure 6, the radiometric and exposure quality of the resulting panorama is far from satisfying, therefore a pre-processing of the raw image data can further improve the results.

The tests performed on the vaults highlighted that the accuracy decrease when frame images match the most deformed areas of the panoramas. To this aim, it would be useful to access the raw pinhole images acquired by the scanner's built-in camera before their stitching. Otherwise, pinhole images can be extracted from the panoramas to reduce deformations, following a procedure as the one described in (Bruno and Roncella, 2019).

A weakness of the presented methodology relies on the quality of the object texture. On homogeneous surfaces the quality of matching degrades and might become troublesome when few frame images are used.

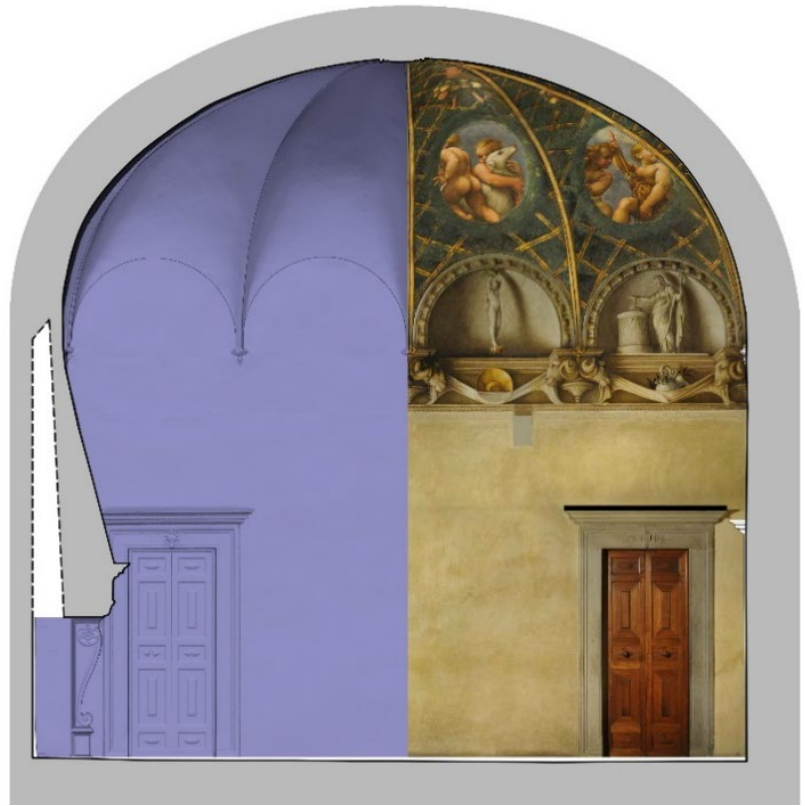

Figure 5. Transversal section of the "Chamber of the Abbess" (on the left: mesh model; on the right: orthophoto).

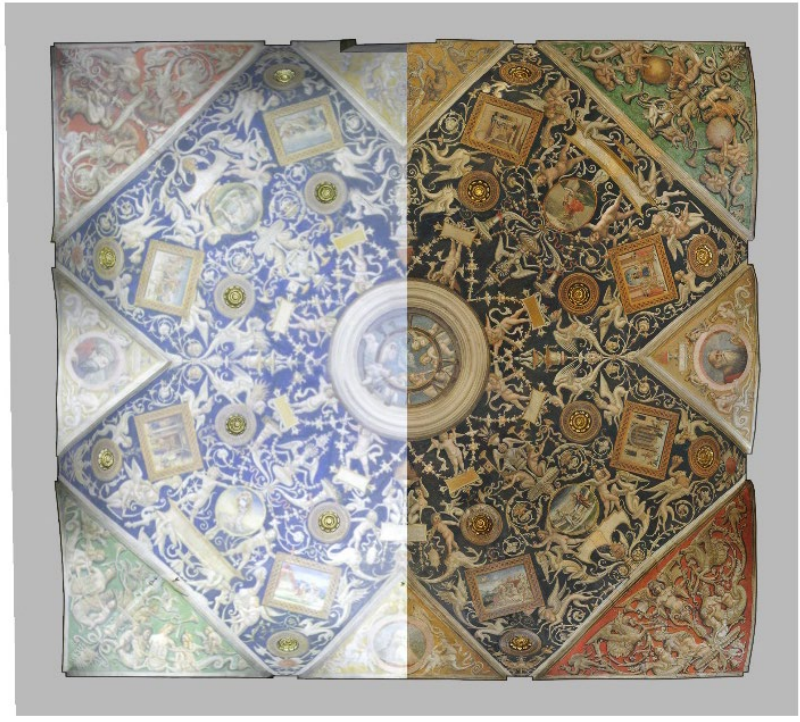

Figure 6. Plan vault of the chamber frescoed by Araldi (on the left: orthophoto computed using the radiometric information provided by the RTC360 camera; on the right: orthophoto computed using the radiometric information provided by the Nikon D3x).

Nonetheless, with good object features, the methodology has proven to be reliable even when the number of frame images is reduced. As long as a minimum overlap between images is guaranteed, the photogrammetric block works and maintains rigidity. It was observed that each tie point must have at least three projections on the frame images to ensure block consistency. Otherwise, the accuracy degrades, being much more influenced by the (usually lower) spherical images resolution.

This flexibility simplifies the data acquisition phase and reduces survey and processing times. The operators can acquire a lower number of images than usually needed in a photogrammetric stand-alone survey, with the certainty of a proper spatial reconstruction provided by the TLS data.

Finally, since it has been observed that using only panoramas as ground reference reduces the internal consistency of the photogrammetric block (reprojection errors are lower using GCP, see Table 2), a further research development could involve the exploitation of geometric data acquired by TLS in addition to the images. In particular, the spatial coordinates of the tie points extracted during the matching could be obtained from the depth maps associated with the panoramas, by projecting them to the panoramas. Then, all the tie points could be constrained as GCP to improve the orientation. In this way, thousands of GCP could be automatically measured, taking benefit from the matching algorithm precision for their detection and measurement on images. In this way the fully integration of range- and imagebased data could be obtained.

\section{CONCLUSIONS}

The paper investigated a procedure that enables the use of a standard photogrammetric image matching procedure to simultaneously process TLS and photogrammetric data. The implemented workflow allowed the correct co-registration of frame images on the spherical images obtained from TLS point cloud, without any GCP but using the automatically extracted tie points and the known EO parameters of the spherical images to set the ground reference. The procedure demonstrated to be reliable even with low overlap between frame images.

The methodology allowed for effective integrated processing 
with the possibility of take benefit from TLS and photogrammetry pros.

The survey campaign carried out allowed to accurately document the museum itinerary known as "Camera di San Paolo" in Parma. The proposed methodology was applied to the entire complex and made it possible to obtain all the two-dimensional drawings required for the restoration project, compatible with a scale of 1:50, and the high-resolution orthophotos $(1 \mathrm{~mm} / \mathrm{pix})$ of all the walls and vaults (Figure 5 and Figure 6).

\section{REFERENCES}

Agisoft Helpdesk Portal, 8/2/2021. Terrestrial laser scanning data processing. https://agisoft.freshdesk.com/support /solutions/arti cles/ 31000159101-terrestrial-laser-scanning-data-processing. (Last access November 2021).

Balletti, C., Guerra, F., Scocca, V., and Gottardi, C., 2015. 3D integrated methodologies for the documentation and the virtual reconstruction of an archaeological site, Int. Arch. Photogramm. Remote Sens. Spatial Inf. Sci., XL-5/W4, 215-222, doi:10.5194/isprsarchives-XL-5-W4-215-2015.

Bay H., Tuytelaars T., Van Gool L., 2006. SURF: Speeded Up Robust Features. In: Leonardis A., Bischof H., Pinz A. (eds) Computer Vision - ECCV 2006. ECCV 2006. Lecture Notes in Computer Science, vol 3951. Springer, Berlin, Heidelberg. https://doi.org/10.1007/11744023_32.

Bianchi, G., Bruno, N., Dall'Asta, E., Forlani, G., Re, C., Roncella, R., Santise, M., Vernizzi, C. and Zerbi, A., 2016. Integrated survey for architectural restoration: a methodological comparison of two case studies, Int. Arch. Photogramm. Remote Sens. Spatial Inf. Sci., XLI (B5), 175-182. https://doi.org/10.5194/isprsarchives-XLI-B5-175-2016.

Bitelli, G., Girelli, V.A., Vannucci, G., Mandanici, E., Pigozzi, M., 2020. Geomatics as a knowledge base propaedeutic to the restoration of an extended fresco wall. In: IMEKO TC4 International Conference on Metrology for Archaeology and Cultural Heritage 2020. Curran Associates, Inc.: Red Hook, pp. 366-371.

Bevilacqua, M.G, Caroti, G., Martínez-Espejo Zaragoza, I., Piemonte, A., 2016: Frescoed Vaults: Accuracy Controlled Simplified Methodology for Planar Development of ThreeDimensional Textured Models. Remote Sensing, 8(3):239. https://doi.org/10.3390/rs8030239.

Bruno, N. and Roncella, R., 2019. Accuracy assessment of 3d models generated from google street view imagery, Int. Arch. Photogramm. Remote Sens. Spatial Inf. Sci., XLII-2/W9, 181188, https://doi.org/10.5194/isprs-archives-XLII-2-W9-1812019,2019

Chiabrando, F., Lingua, A., Noardo, F., Spano, A., 2014: 3D modelling of trompe l'oeil decorated vaults using dense matching techniques. Int. Arch. Photogramm. Remote Sens. Spatial Inf. Sci., II-5, 97-104. https://doi.org/10.5194/isprsannals-II-5-972014.

Fiorillo, F., Limongiello, M., Bolognesi, C., 2021. Image-Based and Range-Based Dataset Integration for an Efficient 3D Representation. In: Arena A., Arena M., Mediati D., Raffa P. (eds) Connecting. Drawing for weaving relationship. Languages Distances Technologies. Proceedings of the 42th International
Conference of Representation Disciplines Teachers. FrancoAngeli: Milano, pp. 2319-2336.

Gabellone, F., Chiffi, M., Tanasi, D., Decker, M., 2020 Integrated Technologies for Indirect Documentation, Conservation and Engagement of the Roman Mosaics of Piazza Armerina (Enna, Italy). In: Cicalò E. (eds) Proceedings of the 2nd International and Interdisciplinary Conference on Image and Imagination. IMG 2019. Advances in Intelligent Systems and Computing, vol. $1140 . \quad$ Springer: Cham. https://doi.org/10.1007/978-3-030-41018-6_83.

Grussenmeyer, P., Alby, E., Landes, T., Koehl, M., Guillemin, S., Hullo, J.-F., Assali, P., and Smigiel, E., 2012. Recording approach of heritage sites based on merging point clouds from high resolution photogrammetry and terrestrial laser scanning. Int. Arch. Photogramm. Remote Sens. Spatial Inf. Sci., XXXIXB5, 553-558.

Hassan, A.T., Fritsch, D., 2019: Integration of Laser Scanning and Photogrammetry in 3D/4D Cultural Heritage Preservation A Review. International Journal of Applied Science and Technology, 9/4, 76-91.

Luhmann, T.; Chizhova, M.; Gorkovchuk, D., 2020. Fusion of UAV and Terrestrial Photogrammetry with Laser Scanning for 3D Reconstruction of Historic Churches in Georgia. Drones 2020, 4, 53. https://doi.org/10.3390/drones4030053.

Muja, M., Lowe, D. G., 2012. Fast Matching of Binary Features. In: Conference on Computer and Robot Vision, pp. 404-410. 10.1109/CRV.2012.60.

Patrucco, G., Chiabrando, F., Dameri, A., Teppati Losè, L., 2020. Geomatic contribution for the restoration project of the Valentino Castle Green Room. From data acquisition to integrated documentation. Int. Arch. Photogramm. Remote Sens. Spatial Inf. Sci., XLIII-B2-2020, 885-893. https://doi.org/10.5194/isprsarchives-XLIII-B2-2020-885-2020.

Remondino F., 2011: Heritage recording and 3D Modeling with Photogrammetry and 3D scanning. Remote Sensing, 3(6), 11041138. https://doi.org/10.3390/rs3061104.

Rönnholm, P., Honkavaara, E., Litkey, P., Hyyppä, H., Hyyppä, J., 2007. Integration of laser scanning and photogrammetry. Int. Arch. Photogramm. Remote Sens. Spatial Inf. Sci., XXXVI3/W52, 355-362.

Rosten, E., Drummond, T., 2005. Fusing Points and Lines for High Performance Tracking. In: Proceedings of the IEEE International Conference on Computer Vision, Vol. 2, pp. 15081511. https://doi.org/10.1109/ICCV.2005.104.

Shi, J., Tomasi, C. 1994. Good Features to Track. In: Proceedings of the IEEE Conference on Computer Vision and Pattern Recognition, pp. 593-600. 10.1109/CVPR.1994.323794.

Valente, R., Oreni, D., Barazzetti, L., Roncoroni, F., Previtali, M., 2019. Documentation of historical architectures through the combined use of digital surveying techniques. Int. Arch. Photogramm. Remote Sens. Spatial Inf. Sci., XLII-2/W11, 11211125. https://doi.org/10.5194/isprs-archives-XLII-2-W11-11212019. 